\title{
CORONA-TYPE THEOREMS AND DIVISION IN SOME FUNCTION ALGEBRAS ON PLANAR DOMAINS
}

\author{
by
}

Raymond Mortini \& Rudolf Rupp

\begin{abstract}
Let $A$ be an algebra of bounded smooth functions on the interior of a compact set in the plane. We study the following problem: if $f, f_{1}, \ldots, f_{n} \in A$ satisfy $|f| \leq \sum_{j=1}^{n}\left|f_{j}\right|$, does there exist $g_{j} \in A$ and a constant $N \in \mathbb{N}$ such that $f^{N}=\sum_{j=1}^{n} g_{j} f_{j}$ ? A prominent role in our proofs is played by a new space, $C_{\bar{\partial}, 1}(K)$, which we call the algebra of $\bar{\partial}$-smooth functions.

In the case $n=1$, a complete solution is given for the algebras $A^{m}(K)$ of functions holomorphic in $K^{\circ}$ and whose first $m$-derivatives extend continuously to $\overline{K^{\circ}}$. This necessitates the introduction of a special class of compacta, the so-called locally L-connected sets.

We also present another constructive proof of the Nullstellensatz for $A(K)$, that is only based on elementary $\bar{\partial}$-calculus and Wolff's method.
\end{abstract}

17.3.2021

\section{Introduction}

Our paper is motivated by the following problem.

Problem 0.1. - Let $A(K)$ be the algebra of all complex-valued functions that are continuous on the compact set $K \subseteq \mathbb{C}$ and holomorphic in the interior $K^{\circ}$ of $K$. Given $f, f_{1}, \ldots, f_{n} \in A(K)$ satisfying

$$
|f| \leq \sum_{j=1}^{n}\left|f_{j}\right|,
$$

does there exist a power $N$ such that $f^{N}$ belongs to the ideal $I_{A(K)}\left(f_{1}, \ldots, f_{n}\right)$ generated by the $f_{j}$ in $A(K)$ ?

2000 Mathematics Subject Classification. - Primary 46J10, Secondary 46J15; 46J20; 30H50.

Key words and phrases. - algebras of analytic functions; ideals; corona-type theorems; Nullstellensatz; division in algebras of smooth functions . 
In view of Wolff's result for the algebra of bounded holomorphic functions in the open unit disk $\mathbb{D}$ (see [2]), the expected value for $N$ seems to be 3 . We are unable to confirm this; our intention therefore is to present sufficient conditions that guarantee the existence of such a constant that may depend on the $n$-tuple $\left(f_{1}, \ldots, f_{n}\right)$. If $f$ is zero-free on $K$ then, by the classical Nullstellensatz for $A(K), f=\sum_{j=1}^{n} f_{j} g_{j}$ for some $g_{j} \in A(K)$, whenever the $f_{j}$ satisfy (0.1). We present a constructive proof of this assertion by using $\bar{\partial}$-calculus. A different constructive proof, indirectly based on properties of the Pompeiu operator

$$
\operatorname{Pf}(z):=\iint_{K} \frac{f(\xi)}{\xi-z} d \sigma(\xi)
$$

and elementary approximation theory, was recently given by the authors in [6].

To achieve our goals, we consider various algebras of smooth functions on $K$. Here is our setting. First we present some notation and introduce, apart from $A(K)$ that was defined above, the spaces we are dealing with.

As usual, if $f_{x}$ and $f_{y}$ are the partial derivatives of $f$, then

$$
\partial f=(1 / 2)\left(f_{x}-i f_{y}\right) \text { and } \bar{\partial} f=(1 / 2)\left(f_{x}+i f_{y}\right)
$$

denote the Wirtinger derivatives of $f$. Let $\mathbb{K}$ be either $\mathbb{R}$ or $\mathbb{C}$.

Definition 0.2. -

(1) $C(K, \mathbb{K})$ is the set of all continuous, $\mathbb{K}$-valued functions on $K$. If $\mathbb{K}=\mathbb{C}$, then we also write $C(K)$ instead of $C(K, \mathbb{C})$

(2) $C^{m}(K)$ is the set of all $f \in C(K)$ that are $m$-times continuously differentiable on $K^{\circ}$ and such that each of the partial derivatives up to the order $m$ extends continuously to $K$;

(3) $A^{m}(K):=A(K) \cap C^{m}(K)$.

Of course $A^{m}(K)$ is the set of all functions $f \in A(K)$ such that $\left.f^{(j)}\right|_{K^{\circ}}$ extends continuously to $K$ for $j=1, \ldots, m$. Finally, we introduce the following space of $\bar{\partial}$-smooth functions. This space will play an important role when solving $\bar{\partial}$-equations.

(4) $C_{\bar{\partial}, 1}(K)$ is the set of all $f \in C(K)$ continuously differentiable in $K^{\circ}$ with $\bar{\partial} f \in C^{1}\left(K^{\circ}\right)$ and such that $\bar{\partial} f$ admits a continuous extension to $K$.

The set of continuous (respectively infinitely often differentiable) functions on $\mathbb{C}$ with compact support is denoted by $C_{c}(\mathbb{C})$ (respectively $C_{c}^{\infty}(\mathbb{C})$ ). If $f \in C(K, \mathbb{K})$, then $\|f\|_{\infty}=\sup _{z \in K}|f(z)|$ and

$$
Z(f)=\{z \in K: f(z)=0\}
$$

is the zero set of $f$.

If $A$ is a commutative unital algebra with unit element denoted by 1 , then the ideal generated by $f_{1}, \ldots, f_{n} \in A$ is denoted by $I_{A}\left(f_{1}, \ldots, f_{n}\right)$; that is

$$
I_{A}\left(f_{1}, \ldots, f_{n}\right)=\left\{\sum_{j=1}^{n} g_{j} f_{j}: g_{j} \in A\right\} .
$$




\section{The algebra of $\bar{\partial}$-smooth functions}

If we endow $C_{\bar{\partial}, 1}(K)$ with the pointwise operations $\left(+, \bullet, \cdot_{s}\right)$, then it becomes an algebra; just note that for $f, g \in C_{\bar{\partial}, 1}(K), \bar{\partial}(f g)=(\bar{\partial} f) g+f \bar{\partial} g$. The most important subalgebra of $C_{\bar{\partial}, 1}(K)$ is $A(K)$. It is obvious that $C_{\bar{\partial}, 1}(K)$ contains all the polynomials in the real variables $x$ and $y$, or what is equivalent, all polynomials in $z$ and $\bar{z}$. In particular $C_{\bar{\partial}, 1}(K)$ is uniformly dense in $C(K)$. Note that the class of real-valued functions $u$ in $C_{\bar{\partial}, 1}(K)$ coincides with $C^{2}\left(K^{\circ}, \mathbb{R}\right) \cap C^{1}(K, \mathbb{R})$, since $2 \bar{\partial} f=u_{x}+i u_{y} \in C^{1}\left(K^{\circ}\right) \cap C(K)$. We point out that for $f \in C_{\bar{\partial}, 1}(K)$, the function $\partial f: K^{\circ} \rightarrow \mathbb{C}$ may not even be bounded. Consider for example on the unit disk $\mathbb{D}$ the standard holomorphic branch of the function $\sqrt{1-z}$. Here

$$
\partial f(z)=f^{\prime}(z)=\frac{1}{2}(1-z)^{-1 / 2},
$$

which is unbounded. Hence

$$
C_{\bar{\partial}, 1}(\overline{\mathbb{D}}) \backslash C^{1}(\overline{\mathbb{D}}) \neq \emptyset \text { and } C^{1}(\overline{\mathbb{D}}) \backslash C_{\bar{\partial}, 1}(D) \neq \emptyset .
$$

On the other hand, $C_{\bar{\partial}, 1}(K)$ is strictly bigger than $C^{2}(K)$. The example above also shows that if $f \in C_{\bar{\partial}, 1}(K)$, then $\bar{f}$ may not belong to $C_{\bar{\partial}, 1}(K)$ (note that $\bar{\partial} \bar{f}=\overline{\partial f}$ ).

Question. Is the restriction algebra $\left.C_{\bar{\partial}, 1}(K)\right|_{K^{\circ}}$ a subalgebra of $C^{2}\left(K^{\circ}\right)$ ? Note that we only assume that $\bar{\partial} f=\frac{u_{x}-v_{y}}{2}+i \frac{u_{y}+v_{x}}{2}$ is continuously differentiable in $K^{\circ}$.

A useful algebraic property is that $C_{\bar{\partial}, 1}(K)$ is inversionally closed; this means that if $f \in C_{\bar{\partial}, 1}(K)$ has no zeros on $K$, then $1 / f \in C_{\bar{\partial}, 1}(K)$.

A generalization to $n$-tuples (=solution to the Bézout equation $\sum_{j=1}^{n} x_{j} f_{j}=1$ ) is given by the following theorem.

Theorem 1.1. - Suppose that the functions $f_{1}, \ldots, f_{n} \in C_{\bar{\partial}, 1}(K)$ have no common zero on $K$. Then the Bézout equation $\sum_{j=1}^{n} x_{j} f_{j}=1$ admits a solution $\left(x_{1}, \ldots, x_{n}\right)$ in $C_{\bar{\partial}, 1}(K)$.

We present two proofs.

Proof. - (1) Let

$$
q_{j}:=\frac{\overline{f_{j}}}{\sum_{k=1}^{n}\left|f_{k}\right|^{2}} .
$$

Then $q_{j} \in C(K)$ and $\sum_{j=1}^{n} q_{j} f_{j}=1$. By Weierstrass' approximation theorem choose a polynomial $p_{j}(z, \bar{z})$ such that on $K$

$$
\left|p_{j}-q_{j}\right| \leq\left(2 \sum_{k=1}^{n}\left\|f_{j}\right\|_{\infty}\right)^{-1}
$$


Then

$$
\left|\sum_{j=1}^{n} p_{j} f_{j}\right| \geq\left|\sum_{j=1}^{n} q_{j} f_{j}\right|-\left|\sum_{j=1}^{n}\left(p_{j}-q_{j}\right) f_{j}\right| \geq 1-\frac{1}{2}=\frac{1}{2} .
$$

Note that $\sum_{j=1}^{n} p_{j} f_{j} \in C_{\bar{\partial}, 1}(K)$. Because $C_{\bar{\partial}, 1}(K)$ is inversionally closed, we get that

$$
x_{j}=\frac{p_{j}}{\sum_{k=1}^{n} p_{k} f_{k}} \in C_{\bar{\partial}, 1}(K) .
$$

Since $\sum_{j=1}^{n} x_{j} f_{j}=1$, we see that $\left(x_{1}, \ldots, x_{n}\right)$ is the desired solution to the Bézout equation in $C_{\bar{\partial}, 1}(K)$.

(2) This is similar to [1, Exercice 25, p. 139]. Let $f_{j}$ be continuously extended to an open neighborhood $U$ of $K$ so that $\sum_{j=1}^{n}\left|f_{j}\right| \geq \delta>0$ on $U$. Let $E_{j}=\left\{z \in U:\left|f_{j}(z)\right|>0\right\}$. Since the functions $f_{j}$ have no common zeros on $U, \bigcup_{j=1}^{n} E_{j}=U$. Let $\left\{\alpha_{j}: j=1, \ldots, n\right\}$ be a $C_{c}^{\infty}$-partition of unity subordinate to the open covering $\left\{E_{1}, \ldots, E_{n}\right\}$ of $K$; that is,

$$
\alpha_{j} \in C_{c}^{\infty}(\mathbb{C}), \quad 0 \leq \alpha_{j} \leq 1, \quad \operatorname{supp} \alpha_{j} \subseteq E_{j}, \quad \sum_{j=1}^{n} \alpha_{j}=1 \text { on } K
$$

(see $\left[\mathbf{9}\right.$, p. 162]). Then $x_{j}:=\alpha_{j} f_{j}^{-1} \in C_{\bar{\partial}, 1}(K)$ and

$$
\sum_{j=1}^{n} x_{j} f_{j}=\sum_{j=1}^{n} \alpha_{j}=1
$$

Next we deal with the generalized Bézout equation $\sum_{j=1}^{n} x_{j} f_{j}=f$.

Proposition 1.2. - Let $f, f_{j} \in C_{\bar{\partial}, 1}(K)$ and suppose that $f$ vanishes in a neighborhood (within $K)$ of $\bigcap_{j=1}^{n} Z\left(f_{j}\right)$. Then $f \in I_{C_{\bar{\partial}, 1}(K)}\left(f_{1}, \ldots, f_{n}\right)$.

Proof. - Let $V \subseteq \mathbb{C}$ be an open set satisfying $\bigcap_{j=1}^{n} Z\left(f_{j}\right) \subseteq V \cap K \subseteq Z(f)$. If $S=K \backslash V$, then $\inf _{S} \sum_{j=1}^{n}\left|f_{j}\right| \geq \delta>0$. As usual we extend the functions $f_{j}$ to continuous functions in $C_{c}(\mathbb{C})$ and denote these extensions by the same symbol. Let $U_{j}=\left\{z \in \mathbb{C}:\left|f_{j}\right|>0\right\}, j=1, \ldots, n$. Then $S \subseteq \bigcup_{j=1}^{n} U_{j}$. Let $\left\{\alpha_{j}: j=1, \ldots, n\right\}$ be a $C_{c}^{\infty}$-partition of unity subordinate to the open covering $\left\{U_{1}, \ldots, U_{n}\right\}$ of $S$. Then $x_{j}:=\alpha_{j} f_{j}^{-1} \in C_{\bar{\partial}, 1}(K)$ and

$$
\sum_{j=1}^{n} x_{j} f_{j}=\sum_{j=1}^{n} \alpha_{j}=1 \text { on } S .
$$

Noticing that $f \equiv 0$ on $K \backslash S$, we get

$$
\sum_{j=1}^{n}\left(f x_{j}\right) f_{j}=f \text { on } K
$$

where $f x_{j} \in C_{\bar{\partial}, 1}(K)$. 


\section{A new proof of the Nullstellensatz for $A(K)$}

Here we give yet another elementary proof of the "Corona Theorem" (or Nullstellensatz) for the algebra $A(K)$ (see [6] for the preceding one). We use Wolff's $\bar{\partial}$-method (see for example [1, p. 130 and p. 139]). Our main tool will be the following well-known theorem (see [1], [8], and [6]). Here $\sigma$ denotes the 2-dimensional Lebesgue measure.

Theorem 2.1. - Let $K \subseteq \mathbb{C}$ be compact, $f \in C(K) \cap C^{1}\left(K^{\circ}\right)$ and let

$$
u(z)=-\frac{1}{\pi} \iint_{K} \frac{f(w)}{w-z} d \sigma(w) .
$$

Then

1. $u \in C(\hat{\mathbb{C}})$,

2. $u \in C^{1}\left(K^{\circ}\right)$ and holomorphic outside $K$,

3. $\bar{\partial} u=f$ on $K^{\circ}$.

Theorem 2.2. - Let $K \subseteq \mathbb{C}$ be a compact set and let $f_{j} \in A(K)$. Then the Bézout equation $\sum_{j=1}^{n} h_{j} f_{j}=1$ admits a solution $\left(h_{1}, \ldots, h_{n}\right)$ in $A(K)$ if and only if the functions $f_{j}$ have no common zero on $K$.

Proof. - Assume that $\sum_{j=1}^{n}\left|f_{j}\right| \geq \delta>0$ on $K$. Applying Theorem 1.1, there is a solution $\left(x_{1}, \ldots, x_{n}\right) \in C_{\bar{\partial}, 1}(K)^{n} \subseteq C(K)^{n}$ to $\sum_{j=1}^{n} x_{j} f_{j}=1$. Now we use Wolff's method to solve a system of $\bar{\partial}$-equations. Consider $\boldsymbol{f}=\left(f_{1}, \ldots, f_{n}\right)$ as a row matrix; its transpose is denoted by $\boldsymbol{f}^{t}$. Let $|\boldsymbol{f}|^{2}=\sum_{j=1}^{n}\left|f_{j}\right|^{2}$; that is $|\boldsymbol{f}|^{2}=\overline{\boldsymbol{f}} \boldsymbol{f}^{t}$.

The Bézout equation now reads as $\boldsymbol{x} \boldsymbol{f}^{t}=1$. It is well-known (see for instance [7, p. 227]) that any other solution $\boldsymbol{u} \in C(K)$ to the Bézout equation $\boldsymbol{u} \boldsymbol{f}^{t}=1$ is given by

$$
\boldsymbol{u}^{t}=\boldsymbol{x}^{t}+H \boldsymbol{f}^{t}
$$

or equivalently

$$
\boldsymbol{u}=\boldsymbol{x}-\boldsymbol{f H},
$$

where $H$ is an $n \times n$ antisymmetric matrix over $C(K)$; that is $H^{t}=-H$.

Let

$$
F=\left(\left(\bar{\partial} \boldsymbol{x}^{t} \cdot \overline{\boldsymbol{f}}\right)^{t}-\bar{\partial} \boldsymbol{x}^{t} \cdot \overline{\boldsymbol{f}}\right) \frac{1}{|\boldsymbol{f}|^{2}} .
$$

Since $\boldsymbol{x} \in C_{\bar{\partial}, 1}(K)^{n}$, we see that $F$ is an antisymmetric matrix over $C(K) \cap C^{1}\left(K^{\circ}\right)$. Thus, by Theorem 2.1, the system $\bar{\partial} H=F$ admits a matrix solution $H$ over $C(K) \cap C^{1}\left(K^{\circ}\right)$. Note that $H$ can be chosen to be antisymmetric, too.

It is now easy to check that on $K^{\circ}, \bar{\partial} \boldsymbol{u}=\mathbf{0}$. In fact

$$
\begin{gathered}
\bar{\partial} \boldsymbol{u}=\bar{\partial} \boldsymbol{x}-\boldsymbol{f} \cdot \bar{\partial} H=\bar{\partial} \boldsymbol{x}-\boldsymbol{f} \cdot\left(\overline{\boldsymbol{f}}^{t} \cdot \bar{\partial} \boldsymbol{x}-\bar{\partial} \boldsymbol{x}^{t} \cdot \overline{\boldsymbol{f}}\right) \frac{1}{|\boldsymbol{f}|^{2}} \\
=\frac{\left(\boldsymbol{f} \cdot \bar{\partial} \boldsymbol{x}^{t}\right) \overline{\boldsymbol{f}}}{|\boldsymbol{f}|^{2}}=\frac{\left(\bar{\partial}\left(\boldsymbol{f} \cdot \boldsymbol{x}^{t}\right)\right) \overline{\boldsymbol{f}}}{|\boldsymbol{f}|^{2}}=\frac{\left(\bar{\partial}\left(\boldsymbol{x} \cdot \boldsymbol{f}^{t}\right)^{t}\right) \overline{\boldsymbol{f}}}{|\boldsymbol{f}|^{2}}=\mathbf{0}
\end{gathered}
$$


Thus $u=\boldsymbol{x}-\boldsymbol{f} H \in A(K)$. Hence $\boldsymbol{u}$ is the solution to the Bézout equation in $A(K)$.

\section{The principal ideal case}

In this section we consider the following division problems: let $A$ be one of the algebras $C^{m}(K), A^{m}(K)$ and $C_{\bar{\partial}, 1}(K)$. Determine the best constant $N \in \mathbb{N}$ such that $|f| \leq|g|$ implies $f^{N} \in I_{A}(g)$; that is $g$ divides $f^{N}$ in $A$. Note that this is the $(n=1)$-case of Problem 0.1.

We need to introduce a special class of compacta.

\section{A. Locally L-connectedness. -}

Definition 3.1. - A compact set $K \subseteq \mathbb{C}$ satisfying $K=\overline{K^{\circ}}$ is said to be locally L-connected if for every $z_{0} \in \partial K$ there is an open neighborhood $U$ of $z_{0}$ in $K$ and a constant $L>0$ such that every point $z \in U \cap K^{\circ}$ can be joined with $z_{0}$ by a piecewise $C^{1}$-path $\gamma_{z}$ entirely contained in $K^{\circ}$ (except for the end-point $z_{0}$ ) and such that

$$
L\left(\gamma_{z}\right) \leq L\left|z-z_{0}\right|
$$

where $L\left(\gamma_{z}\right)$ denotes the length of the path $\gamma_{z}$.

As examples we mention closed disks, compact convex sets with interior, and finite unions of these sets. Counterexamples, for instance, are comb domains, spirals having infinite length and certain disjoint unions of infinitely many disks (see below).

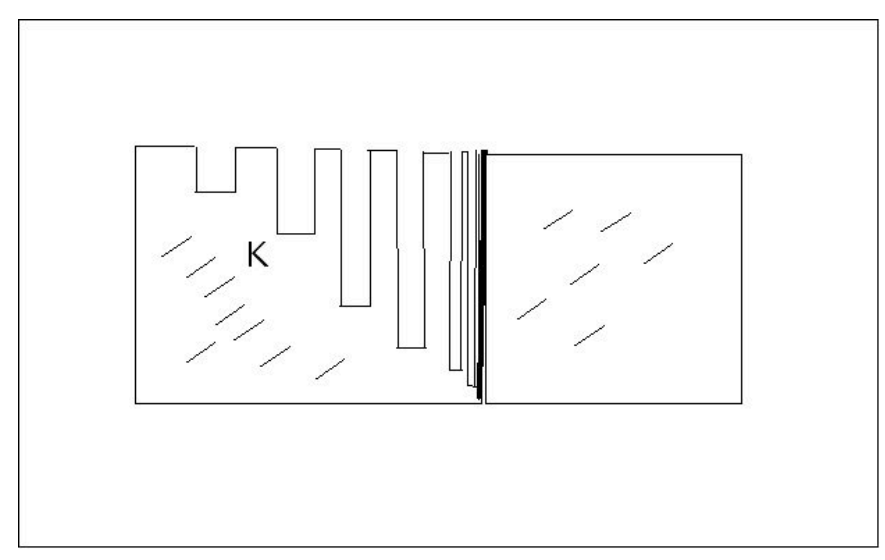

Figure 1. The comb domain

Definition 3.2. - A topological space $X$ is said to be locally path-connected if for every $x \in X$ and every neighborhood $U \subseteq X$ of $x$ there exists a neighborhood $V$ of $x$ such that $V \subseteq U$ and for any pair $(u, v)$ of points in $V$ there is a path from $u$ to $v$ lying in $U$. (1)

\footnotetext{
(1) In the "usual" definition the path is assumed to lie in $V$; these two definitions coincide
} 


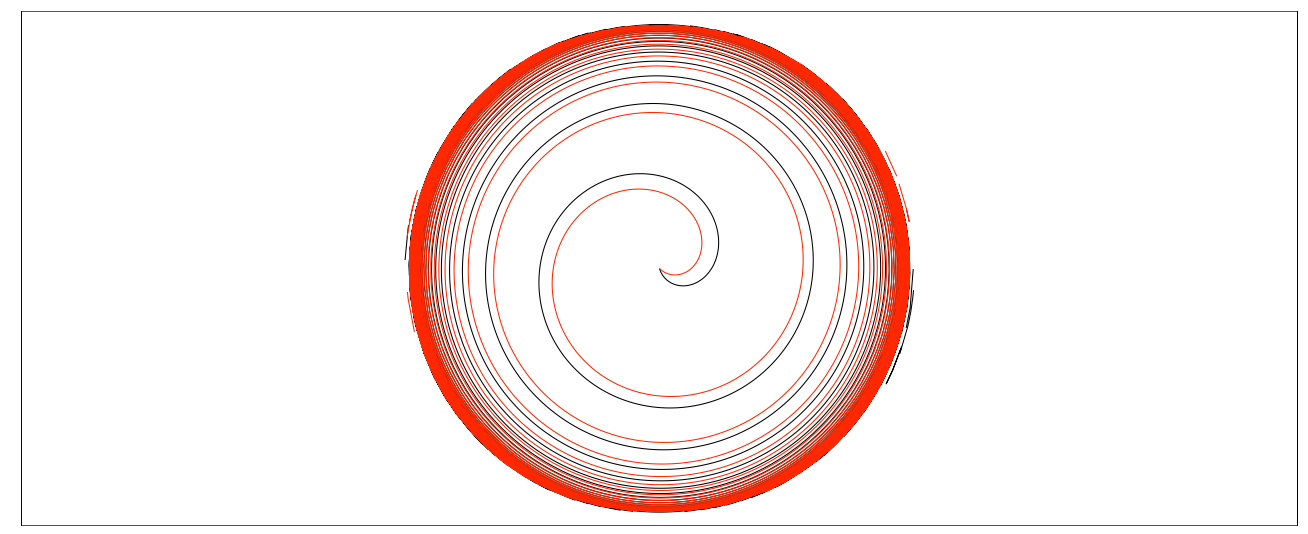

Figure 2. The spiral domain

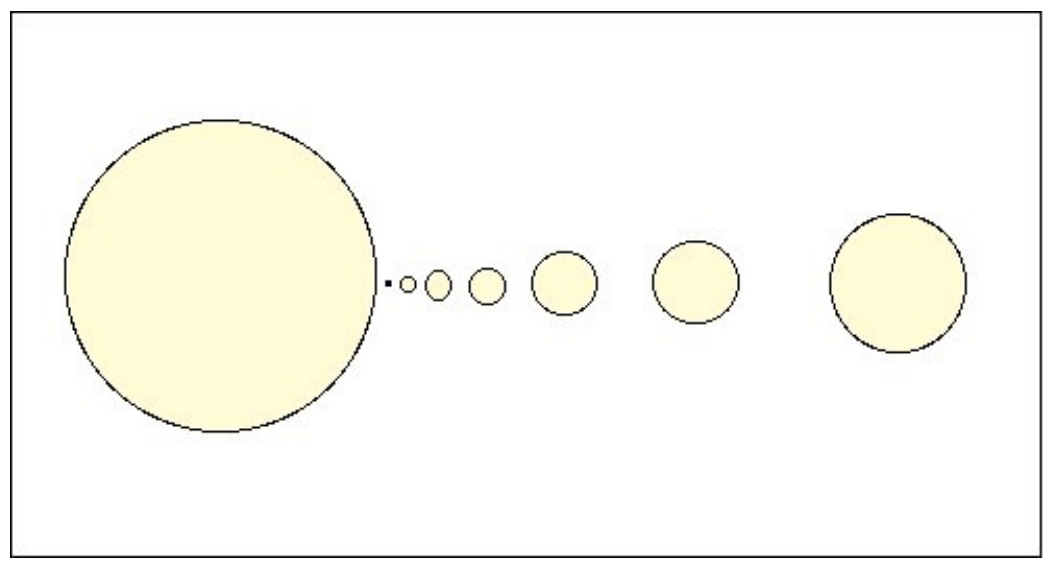

Figure 3. A non locally L-connected compactum

Proposition 3.3. - Every locally L-connected compactum is locally path-connected.

Proof. - Let $z_{0} \in \partial K=\overline{K^{\circ}} \backslash K^{\circ}$. Given the open disk $D\left(z_{0}, r\right)$, we choose the open neighborhood $U$ of $z_{0}$ in $K$ and $L=L\left(z_{0}\right)>0$ according to the definition of local L-connectivity. In particular, every point $z \in D\left(z_{0}, r\right) \cap U \cap K^{\circ}$ can be joined with $z_{0}$ by a curve $\gamma_{z, z_{0}}$ contained in $K^{\circ} \cup\left\{z_{0}\right\}$. Note that for $z \in U \cap K^{\circ}, \gamma_{z, z_{0}} \subseteq D\left(z_{0}, L\left|z-z_{0}\right|\right)$. Hence, if we choose $0<r^{\prime}<r / 2 L$ so small that $D\left(z_{0}, r^{\prime}\right) \cap K \subseteq U$, then every point $z \in D\left(z_{0}, r^{\prime}\right) \cap K^{\circ}$ can be joined with $z_{0}$ by a path $\gamma_{z, z_{0}}$ contained in

$$
K \cap D\left(z_{0}, L\left|z-z_{0}\right|\right) \subseteq K \cap D\left(z_{0}, r\right) .
$$

We still need to show that every point $z_{1} \in \partial K \cap D\left(z_{0}, r^{\prime}\right)$ can be joined with $z_{0}$ by a path contained in $K \cap D\left(z_{0}, r\right)$. By the same argument as above, there is a disk $D\left(z_{1}, r^{\prime \prime}\right) \subseteq D\left(z_{0}, r^{\prime}\right)$ such that 
every point $z \in D\left(z_{1}, r^{\prime \prime}\right) \cap K^{\circ}$ can be joined with $z_{1}$ by a curve $\gamma_{z, z_{1}}$ contained in

$$
K \cap D\left(z_{1}, L\left(z_{1}\right) r^{\prime \prime}\right) \subseteq K \cap D\left(z_{0}, r\right) .
$$

Thus the concatenation of the inverse path $\gamma_{z, z_{1}}^{-1}$ with $\gamma_{z, z_{0}}$ joins $z_{1}$ with $z_{0}$ within $D\left(z_{0}, r\right) \cap K$. Hence every point $w \in D\left(z_{0}, r^{\prime}\right) \cap K$ can be joined to $z_{0}$ by a path contained in $D\left(z_{0}, r\right) \cap K$. If $z_{0} \in K^{\circ}$, then the assertion is trivial.

We conclude that for $z_{0} \in K$ and $D\left(z_{0}, r\right)$ we find a neighborhood $V$ of $z_{0}$ in $K$ such that any two points $u, v \in V$ can be joined by a path (passing through $z_{0}$ ) that stays in $D\left(z_{0}, r\right)$. Hence $K$ is locally path-connected.

We mention that there exist locally connected, path-connected continua $K$ with $\overline{K^{\circ}}=K$ that are not locally L-connected. For example, let $K$ be the inner spiral $(\theta+1)^{-1} \leq r(\theta) \leq \theta^{-1}, \pi \leq \theta \leq \infty$. See the following figure. Here the point $0 \in \partial K$, but cannot be joined with any other point $z \in K$ by a rectifiable path. We can also take a spiral consisting of "thick" half-circles with radii $1 / n$, $n=1,2, \ldots$
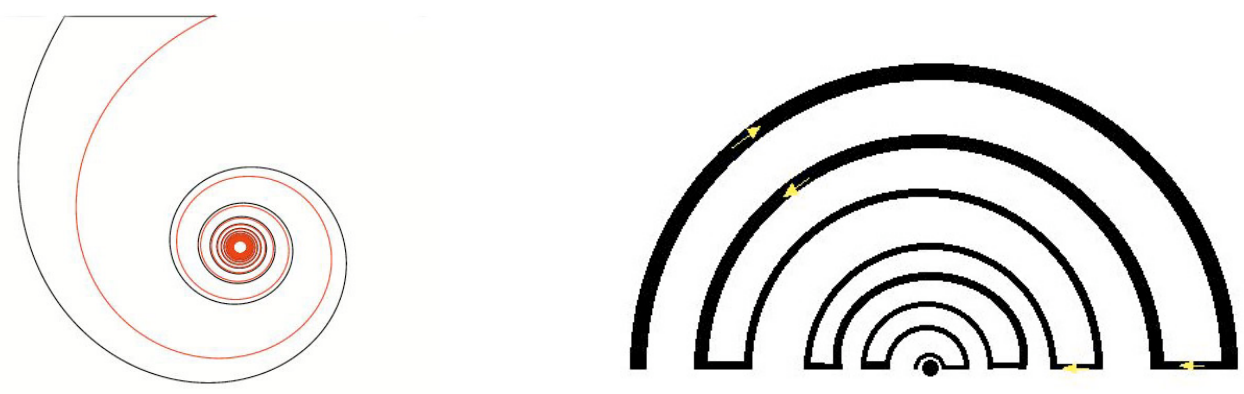

3.B. The Taylor formula on the boundary. - We need the following Taylor formula for functions in $A^{m}(K)$.

Proposition 3.4. - (1) Let $K$ be a locally L-connected compactum. Given $f \in A^{m}(K)$ and $z_{0} \in \overline{K^{\circ}} \backslash K^{\circ}=\partial K$, we denote the continuous extension of $f^{(j)}$ to $z_{0}$ by the symbol $f^{(j)}\left(z_{0}\right)$, $(j=0,1, \ldots, m)$. Let

$$
P_{m}\left(z, z_{0}\right)=f\left(z_{0}\right)+\frac{1}{1 !} f^{\prime}\left(z_{0}\right)\left(z-z_{0}\right)+\cdots+\frac{1}{m !} f^{(m)}\left(z_{0}\right)\left(z-z_{0}\right)^{m}
$$

be the $m$-th Taylor polynomial of $f$ at $z_{0}$. Then

$$
f(z)=P_{m}\left(z, z_{0}\right)+R_{m}(z),
$$

where $R_{m} \in A^{m}(K), R_{m}^{(j)}\left(z_{0}\right)=0$ for $j=0,1, \ldots, m$ and

$$
R_{m}^{(j)}(z)=\mathcal{O}\left(z-z_{0}\right)^{m-j} \text { as } z \rightarrow z_{0} .
$$


Moreover,

$$
\lim _{\substack{z \rightarrow z_{0} \\ z \in K^{\circ}}} f^{\prime}(z)=\lim _{\substack{z \rightarrow z_{0} \\ z \in K \backslash\left\{z_{0}\right\}}} \frac{f(z)-f\left(z_{0}\right)}{z-z_{0}} .
$$

(2) If $K$ is not locally L-connected, then the equality (3.1) does not hold in general.

Proof. - (1) We first show equality (3.1). Set $\ell:=\lim _{\substack{z \rightarrow z_{0} \\ z \in K^{\circ}}} f^{\prime}(z)$. Let $U \subseteq K$ be the neighborhood of $z_{0}$ and $L>0$ the associated constant given by the definition 3.1 of locally L-connectedness.

Given $\varepsilon>0$, choose $\delta>0$ so small that $\left|f^{\prime}(\xi)-\ell\right|<\varepsilon / L$ for $\left|\xi-z_{0}\right|<\delta, \xi \in K^{\circ}$. For $z \in D\left(z_{0}, \delta / L\right) \cap U \cap K^{\circ}$, let $\gamma_{z, z_{0}}$ be a path in $K^{\circ} \cup\left\{z_{0}\right\}$ joining $z$ with $z_{0}$ and with length $L\left(\gamma_{z, z_{0}}\right) \leq L\left|z-z_{0}\right|$. Note that

$$
\gamma_{z, z_{0}} \subseteq D\left(z_{0}, L\left|z-z_{0}\right|\right) \subseteq D\left(z_{0}, \delta\right) .
$$

Then, by integrating along $\gamma_{z, z_{0}}$ from $z$ to a point $\tilde{z}_{0}$ close to $z_{0}$ and passing to the limit, we obtain

$$
f(z)-f\left(z_{0}\right)=\int_{\gamma_{z, z_{0}}} f^{\prime}(\xi) d \xi
$$

Hence

$$
\begin{aligned}
\left|\frac{f(z)-f\left(z_{0}\right)}{z-z_{0}}-\ell\right| & \leq \frac{1}{\left|z-z_{0}\right|} \int_{\gamma_{z, z_{0}}}\left|f^{\prime}(\xi)-\ell\right||d \xi| \\
& \leq \frac{1}{\left|z-z_{0}\right|} \frac{\varepsilon}{L} L\left(\gamma_{z, z_{0}}\right) \leq \varepsilon .
\end{aligned}
$$

This confirms (3.1).

Since $f \in A^{m}(K)$, we obviously have $R_{m} \in A^{m}(K)$. Moreover, by taking derivatives in $K^{\circ}$ and extending them to the boundary, we see that $R_{m}^{(j)}\left(z_{0}\right)=\left(f-P_{m}\right)^{(j)}\left(z_{0}\right)=0$ for $j=0,1, \ldots, m$. Moreover, $R_{m}^{(m)}(z)=\mathcal{O}(1)$ for $z \rightarrow z_{0}$. As in the proof of (3.1), for $z \in K^{\circ} \cap U$,

$$
R_{m}^{(m-1)}(z)=\int_{\gamma_{z, z_{0}}} R_{m}^{(m)}(\xi) d \xi
$$

Hence,

$$
\left|R_{m}^{(m-1)}(z)\right| \leq L\left|z-z_{0}\right| \max _{\xi \in \gamma_{z, z_{0}}}\left|R_{m}^{(m)}(\xi)\right|=\mathcal{O}\left(z-z_{0}\right)
$$

Also,

$$
\begin{aligned}
R_{m}^{(m-2)}(z) & =\int_{\gamma_{z, z_{0}}} R_{m}^{(m-1)}(\xi) d \xi \\
& =\int_{\gamma_{z, z_{0}}}\left(\xi-z_{0}\right) \frac{R_{m}^{(m-1)}(\xi)}{\xi-z_{0}} d \xi
\end{aligned}
$$


and so

$$
\begin{aligned}
\left|R_{m}^{(m-2)}(z)\right| & \leq \max _{\xi \in \gamma_{z, z_{0}}} \frac{\left|R_{m}^{(m-1)}(\xi)\right|}{\left|\xi-z_{0}\right|} \int_{\gamma_{z, z_{0}}}\left|\xi-z_{0}\right||d \xi| \\
& \leq \mathcal{O}(1) L\left(\gamma_{z, z_{0}}\right)^{2}=\mathcal{O}\left(z-z_{0}\right)^{2} .
\end{aligned}
$$

Using backward induction, we obtain the assertions that for $j=m, m-1, \ldots, 1,0$

$$
R_{m}^{(j)}(z)=\mathcal{O}\left(z-z_{0}\right)^{m-j}
$$

as $z \rightarrow z_{0}$.

(2) Let $K$ be the union of the disk $D_{0}=\{z:|z+1| \leq 1\}$ and the disks

$$
D_{n}=\left\{z:|z-1 / n| \leq 1 / n^{3}\right\}, n=3,4, \ldots \text {. }
$$

Note that $D_{n} \cap D_{m}=\emptyset$ for $n \neq m, n, m \geq 3$, and that $K=\overline{K^{\circ}}$, but that $K$ is not locally path-connected (at the origin). Let $f$ be defined as follows: $f=0$ on $D_{0}$ and $f=1 / \sqrt{n}$ on $D_{n}$. Then $f$ is continuous on $K$, holomorphic on $K^{\circ}$ and $f^{\prime} \equiv 0$ on $K^{\circ}$. It is obvious that $f^{\prime}$ admits a continuous extension to $K$, namely by the constant 0 . However,

$$
\frac{f(1 / n)-f(0)}{1 / n-0}=\sqrt{n} \rightarrow \infty .
$$

Thus the continuous extension of $f^{\prime}$ to 0 is distinct from the limit of the associated differential quotient:

$$
0 \neq \lim _{\substack{z \rightarrow 0, z \in K^{\circ} \\ \operatorname{Re} z>0}} \frac{f(z)-f(0)}{z-0}=\infty .
$$

Corollary 3.5. - Let $f \in A^{1}(K)$, where $K$ is a locally L-connected compactum. Then for every

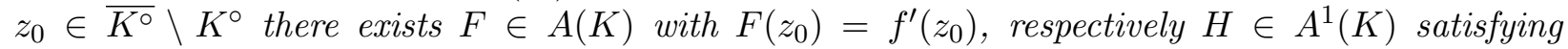
$H\left(z_{0}\right)=H^{\prime}\left(z_{0}\right)=0$ and $H(z)=\mathcal{O}\left(z-z_{0}\right)$, such that

$$
\begin{aligned}
f(z) & =f\left(z_{0}\right)+\left(z-z_{0}\right) f^{\prime}\left(z_{0}\right)+H(z) \\
& =f\left(z_{0}\right)+\left(z-z_{0}\right) F(z)
\end{aligned}
$$

Remark The function $F$ in the representation $f(z)=f\left(z_{0}\right)+\left(z-z_{0}\right) F(z)$ for $f \in A^{1}(K)$ may not belong to $A^{1}(K)$ itself. In fact, let $f(z)=(1-z)^{3} S(z), z \in \mathbb{D}$, where

$$
S(z)=\exp \left(-\frac{1+z}{1-z}\right)
$$

is the atomic inner function. Then $f \in A^{1}(\overline{\mathbb{D}})$ and $f(z)=(1-z) F(z)$ with $F(z)=(1-z)^{2} S(z)$. But $F \notin A^{1}(\overline{\mathbb{D}})$. 
3.C. Division in $A^{m}(K)$. - We first consider the cases $m=0,1$.

\section{Theorem 3.6. -}

a) If $f, g \in C(K)$ satisfy $|f| \leq|g|$, then $f^{2} \in I_{C(K)}(g)$.

b) If $f, g \in C^{1}(K)$ satisfy $|f| \leq|g|$ and if $Z(g)$ has no cluster points in $K^{\circ}$, then $f^{3} \in I_{C^{1}(K)}(g)$.

c) If $f, g \in A(K)$ satisfy $|f| \leq|g|$, then $f^{2} \in I_{A(K)}(g)$.

d) If $f, g \in A^{1}(K)$ satisfy $|f| \leq|g|$, then $f^{3} \in I_{A^{1}(K)}(g)$.

e) If $f, g \in A^{1}(K)$ satisfy $|f| \leq|g|$, then $f^{2} \in I_{A^{1}(K)}(g)$ whenever $K$ is locally L-connected.

f) If $f, g \in C_{\bar{\partial}, 1}(K)$ satisfy $|f| \leq|g|$, and if $Z(g)$ has no cluster points in $K^{\circ}$, then $f^{4} \in$ $I_{C_{\bar{\partial}, 1}(K)}(g)$.

The powers 2,3,2,3,2,4 (within $\mathbb{N}$ ) are optimal.

Proof. - a) Since the quotient $f / g$ is bounded on $K \backslash Z(g)$, we just have to put $h=f^{2} / g$ on $K \backslash Z(g)$ and $h=0$ on $Z(g)$ in order to see that $f^{2}=g h$, where $h \in C(K)$. In fact, if $z \in \partial Z(g)$, then $g(z)=0$ and so $|f| \leq|g|$ implies $f(z)=0$, too. Hence, for any sequence $z_{n}$ in $K \backslash Z(g)$ with $z_{n} \rightarrow z_{0}$ we see that $f^{2} / g\left(z_{n}\right) \rightarrow 0$. Thus $h \in C(K)$ and so $f^{2} \in I_{C(K)}(g)$.

b) Let $h=f^{3} / g$ on $K \backslash Z(g)$ and $h=0$ on $Z(g)$. Then by a), $h \in C(K)$ and $h \in C^{1}(K \backslash Z(g))$. Let $D$ be one of the derivatives $d / d x$ or $d / d y$. Then

$$
D h=\frac{3 g f^{2}(D f)-f^{3}(D g)}{g^{2}} \text { on } K^{\circ} \backslash Z(g) \text {. }
$$

Because $f, g \in C^{1}(K),\left.D f\right|_{K^{\circ}}$ and $\left.D g\right|_{K^{\circ}}$ extend continuously to $\overline{K^{\circ}}$. So $\left.D h\right|_{K^{\circ} \backslash Z(g)}$ extends continuously to $\overline{K^{\circ} \backslash Z}(g)$. Moreover, the assumption $|f| \leq|g|$ implies that $|D h| \leq \kappa|g|$ on $K^{\circ} \backslash Z(g)$. Hence, $\left.D h\right|_{K^{\circ} \backslash Z(g)}$ extends continuously to $\overline{K^{\circ}} \cap Z(g)$ (with value 0 ). By combining both facts, we conclude that $\left.D h\right|_{K^{\circ} \backslash Z(g)}$ extends continuously to $\overline{K^{\circ}}$. Tietze's theorem now yields the extension of $\left.D h\right|_{K^{\circ}}$ to a continuous function on $K$.

If $a \in Z(g) \cap K^{\circ}$ is an isolated point then, by the mean value theorem, $D h(a)$ exists and coincides with the continuous extension of $\left.D h\right|_{K \backslash Z(g)}$ at $a$. Thus $h \in C^{1}\left(K^{\circ}\right)$.

Putting it all together, we conclude that $h \in C^{1}(K)$. Hence $f^{3}=g h \in I_{C^{1}(K)}(g)$.

We remark that if $Z(g) \cap K^{\circ}$ is not discrete, then we are not always able to conclude that $h$ is differentiable at points in $\overline{K^{\circ} \backslash Z(g)} \cap Z(g) \cap K^{\circ}$. (2)

c) If $f, g \in A(K)$, then $|f| \leq|g|$ implies that every zero of $g$ is a zero of $f$. By Riemann's singularity theorem, the boundedness of the quotient $f / g$ around isolated zeros of $g$ within $K^{\circ}$

\footnotetext{
${ }^{(2)}$ Note that the possibility of a continuous extension of the partial derivatives to $E=Z(g)$ does not mean that the function itself is differentiable at the points in $E$. See Proposition 3.4(2) or just consider the Cantor function $C$ associated with the Cantor set $E$ in $[0,1]$. Here the derivative of $C$ is zero at every point in $\mathbb{R} \backslash E, C(0)=0$, $C(1)=1, C$ increasing, but $C$ itself does not belong to $C^{1}(\mathbb{R})$.
} 
implies that $f / g$, and hence $f^{2} / g$, are holomorphic. If $g$ is constantly zero on a component $\Omega_{0}$ of $K^{\circ}$, then $f \equiv 0$ on $\Omega_{0}$, too. So we have just to define $f^{2} / g=0$ there. Using a) and the fact that $\partial\left(Z(g)^{\circ}\right) \subseteq \partial K$, we conclude that

$$
h(z)= \begin{cases}\frac{f^{2}(z)}{g(z)} & \text { if } z \in K \backslash Z(g) \\ 0 & \text { if } z \in Z(g)\end{cases}
$$

belongs to $A(K)$. Hence $f^{2} \in I_{A(K)}(g)$.

d) Let $S=K \backslash Z(g)^{\circ}$. Since the zeros of the holomorphic function $g$ do not accumulate at any points in $S^{\circ}$, we deduce from b) that on $S, f^{3}=k g$ for some $k \in C^{1}(S)$. Moreover, $k=0$ and $D k=0$ on $Z(g) \cap S$.

If we let $h=k$ on $S$ and $h=0$ on $Z(g)$, then $h \in C(K)$. Let

$$
K^{\circ}=\left(\bigcup_{n} \Omega_{n}\right) \cup\left(\bigcup_{j} \Omega_{j}^{\prime}\right)
$$

where the $\Omega_{n}$ are those components of $K^{\circ}$ containing only isolated zeros of $g$ and where the $\Omega_{j}^{\prime}$ are those components of $K^{\circ}$ where $g$ vanishes identically. Note that $\Omega_{n}$ and $\Omega_{j}^{\prime}$ are open sets. Since the quotient $f^{3} / g$ extends holomorphically at every isolated zero of $g$, we conclude that $h$ is holomorphic on each of these components. Hence $h \in A(K)$. Moreover,

$$
\text { (3) } \partial K=\partial S \cup \partial\left(\bigcup_{j} \Omega_{j}^{\prime}\right)
$$

Since $k \in C^{1}(S)$ and $D h=0$ on $\bigcup_{j} \Omega_{j}^{\prime}$, we deduce that $D h$ has a continuous extension to $\partial K$. Consequently, $h \in A^{1}(K)$.

e) Let $f, g \in A^{1}(K)$. Define $h$ by

$$
h(z)= \begin{cases}\frac{f^{2}(z)}{g(z)} & \text { if } z \in K \backslash Z(g) \\ 0 & \text { if } z \in Z(g) .\end{cases}
$$

Then, by c), $h \in A(K)$. Since $K$ is locally L-connected, and so $K=\overline{K^{\circ}}$, we may apply Corollary 3.5. Hence, for every $z_{0} \in \partial K$ there exists $F, G \in A(K)$ such that $f(z)=f\left(z_{0}\right)+\left(z-z_{0}\right) F(z)$ and $g(z)=g\left(z_{0}\right)+\left(z-z_{0}\right) G(z)$. In particular, if $g\left(z_{0}\right)=0,|f| \leq|g|$ implies that $f\left(z_{0}\right)=0$ and $|F| \leq|G|$. If additionally $g^{\prime}\left(z_{0}\right)=0$, then $G\left(z_{0}\right)=F\left(z_{0}\right)=0$; hence $f^{\prime}\left(z_{0}\right)=0$, too. Now, for

$\overline{{ }^{(3)} \text { Actually we have } \partial\left(Z(g)^{\circ}\right) \subseteq \partial S=\partial K}$ 
$z \in K^{\circ} \backslash Z(g)$,

$$
\begin{aligned}
\partial\left(\frac{f^{2}(z)}{g(z)}\right) & =\frac{2 f(z) g(z) f^{\prime}(z)-f^{2}(z) g^{\prime}(z)}{g^{2}(z)} \\
& =2 \frac{f(z)}{g(z)} f^{\prime}(z)-\left(\frac{f(z)}{g(z)}\right)^{2} g^{\prime}(z) .
\end{aligned}
$$

Case 1 Let $z_{0} \in \overline{K \backslash Z(g)} \cap Z(g)$. This means that $z_{0}$ is in the boundary of $Z(g)$ with respect to the topological space $K$. Note also that by our global assumption, $z_{0} \in \partial K$.

1.1 If $g^{\prime}\left(z_{0}\right)=f^{\prime}\left(z_{0}\right)=0$, then, by (3.4), the boundedness of the quotient $\left.(f / g)\right|_{\overline{K^{\circ}} \backslash Z(g)}$ implies that $\left.\left(\partial\left(\frac{f^{2}(z)}{g(z)}\right)\right)\right|_{\overline{K^{\circ}} \backslash Z(g)}$ admits a continuous extension to $z_{0}$.

1.2 If $g^{\prime}\left(z_{0}\right) \neq 0$, then $G\left(z_{0}\right) \neq 0$. Hence

$$
\frac{f(z)}{g(z)}=\frac{\left(z-z_{0}\right) F(z)}{\left(z-z_{0}\right) G(z)}=\frac{F(z)}{G(z)},
$$

is continuous at $z_{0}$. Thus, by (3.4), the continuity of $f^{\prime}$ and $g^{\prime}$ implies that $\left.\left(\partial\left(\frac{f^{2}(z)}{g(z)}\right)\right)\right|_{\overline{K^{\circ}} \backslash Z(g)}$ admits a continuous extension to $z_{0}$.

Case 2 Let $z_{0} \in \operatorname{int} Z(g) \cap \partial K$, the interior being taken in the topological space $K$. Then there is an open set $U$ in $\mathbb{C}$ containing $z_{0}$ such that $U \cap K \subseteq Z(g)$. Since $K=\overline{K^{\circ}}, V:=U \cap K^{\circ} \neq \emptyset$. Moreover $g \equiv 0$ on $V$. Note that by the definition of $h,\left.\left(f^{2} / g\right)\right|_{\overline{K^{\circ}} \backslash Z(g)}$ has a continuous extension to $K$ with values 0 on $Z(g)$. Thus the derivative of $h$ is zero on $V$, and the derivative of the null-function $\left.h\right|_{V}$ has a continuous extension to $z_{0}$ with value 0 .

Combining both cases, we conclude that $h \in A^{1}(K)$ and so $f^{2} \in I_{A^{1}(K)}(g)$.

f) Let $h=f^{4} / g$ on $K \backslash Z(g)$ and $h=0$ on $Z(g)$. Using b), we know that $h=\left.f\left(f^{3} / g\right)\right|_{K^{\circ} \backslash Z(g)}$ admits an extension to a function in $C^{1}\left(K^{\circ}\right)$. (Here we have used that the zeros of $g$ are isolated in $\left.K^{\circ}\right)$. Next we have to consider the second-order derivatives. Since on $K^{\circ} \backslash Z(g)$

$$
\bar{\partial} h=\frac{4 g(\bar{\partial} f) f^{3}-f^{4} \bar{\partial} g}{g^{2}}
$$

we obtain

$$
\partial(\bar{\partial} h)=\frac{g^{2}\left[4(\partial g)(\bar{\partial} f) f^{3}+4 g(\partial \bar{\partial} f) f^{3}+12 g(\bar{\partial} f) f^{2}(\partial f)-4 f^{3}(\partial f)(\bar{\partial} g)-f^{4}(\partial \bar{\partial} g)\right]-8 g^{2}(\bar{\partial} f) f^{3}(\partial g)+2 f^{4} g(\bar{\partial} g)(\partial g)}{g^{4}}
$$

and

$$
\bar{\partial}(\bar{\partial} h)=\frac{g^{2}\left[4(\bar{\partial} g)(\bar{\partial} f) f^{3}+4 g(\bar{\partial} \bar{\partial} f) f^{3}+12 g(\bar{\partial} f)^{2} f^{2}-4 f^{3}(\bar{\partial} f)(\bar{\partial} g)-f^{4}(\bar{\partial} \bar{\partial} g)\right]-8 g^{2}(\bar{\partial} f) f^{3}(\bar{\partial} g)+2 f^{4} g(\bar{\partial} g)^{2}}{g^{4}} .
$$

Using that $|f| \leq|g|$ we conclude from (3.6) that on $K^{\circ} \backslash Z(g)$,

$$
|\bar{\partial} h| \leq 4|\bar{\partial} f||g|^{2}+|\bar{\partial} g||g|^{2} \leq C|g|^{2} .
$$

Hence $\left.\bar{\partial} h\right|_{K^{\circ} \backslash Z(g)}$ admits a continuous extension to $\overline{K^{\circ}}$ with value 0 on $Z(g) \cap \overline{K^{\circ}}$. Using Tietze's theorem we get a continuous extension to $K$, too. 
To show that $\bar{\partial} h$ is in $C^{1}\left(K^{\circ}\right)$, we use the formulas above to conclude that for every $z_{0} \in$ $\overline{K^{\circ} \backslash Z(g)} \cap K^{\circ}$ there is a small neighborhood $U$ of $z_{0}$ such that on $U \backslash Z(g)$

$$
\max \{|\bar{\partial}(\bar{\partial} h)|,|\partial(\bar{\partial} h)|\} \leq C|g| .
$$

Thus $\left.\bar{\partial}(\bar{\partial} h)\right|_{K^{\circ} \backslash Z(g)}$ and $\left.\partial(\bar{\partial} h)\right|_{K^{\circ} \backslash Z(g)}$ admit continuous extensions to $K^{\circ}$ with value 0 . Since the zeros of $g$ within $K^{\circ}$ are isolated, we deduce that $\left.\bar{\partial} h\right|_{K^{\circ} \backslash Z(g)}$ belongs to $C^{1}\left(K^{\circ}\right)$.

Thus $h \in C_{\bar{\partial}, 1}(K)$ and so $f^{4} \in I_{C_{\bar{\partial}, 1}(K)}(g)$.

To show the optimality of the powers, let $K$ be the closed unit disk $\overline{\mathbb{D}}$ and consider for a) and c) the functions $f(z)=(1-z) S(z)$ and $g(z)=1-z$ where

$$
S(z)=\exp \left(-\frac{1+z}{1-z}\right)
$$

is the atomic inner function. Then on $\mathbb{D}, f / g=S$ and $|f| \leq|g|$. But $S$ does not admit a continuous extension to $\overline{\mathbb{D}}$.

For b), let $f(z)=z$ and $g(z)=\bar{z}$. Then the $\bar{\partial}$-derivative of $f^{2} / g$ outside 0 does not admit a continuous extension to 0 ; in fact, $\bar{\partial}\left(f^{2}(z) / g(z)\right)=\bar{\partial}\left(z^{2} / \bar{z}\right)=-z^{2} / \bar{z}^{2}$, a function that is discontinuous at 0 .

For e) let $f(z)=(1-z)^{3} S(z)$ and $g(z)=(1-z)^{3}$. Then $f, g \in A^{1}(\overline{\mathbb{D}}),|f| \leq|g|$ but $f / g \notin A^{1}(\overline{\mathbb{D}})$.

For $\mathrm{f})$ Let $f(z)=z$ and $g(z)=\bar{z}$. Then the $\partial$-derivative of $\bar{\partial}\left(f^{3} / g\right)$ outside 0 does not admit a continuous extension to 0 ; in fact,

$$
\bar{\partial}\left(f^{3}(z) / g(z)\right)=\bar{\partial}\left(z^{3} / \bar{z}\right)=-z^{3} / \bar{z}^{2} .
$$

Hence

$$
\partial\left(\bar{\partial}\left(f^{3}(z) / g(z)\right)\right)=-\partial\left(z^{3} / \bar{z}^{2}\right)=-3 z^{2} / \bar{z}^{2} .
$$

For d) we necessarily have to consider a compactum that is not locally L-connected. Moreover, as the proof of e) shows, the extensions of $\left.f^{\prime}\right|_{K^{\circ} \backslash Z(g)}$ and $\left.g^{\prime}\right|_{K^{\circ} \backslash Z(g)}$ to a point $z_{0} \in \partial K \cap Z(g)$ cannot both be zero. As an example we take the following compact set $K$ (see figure 4 ):

$$
K=\{0\} \cup \bigcup_{n=1}^{\infty}\left\{z \in \mathbb{C}: \frac{1}{2^{2 n+1}} \leq|z| \leq \frac{1}{2^{2 n}}:|\arg z| \leq \pi / 4\right\} .
$$




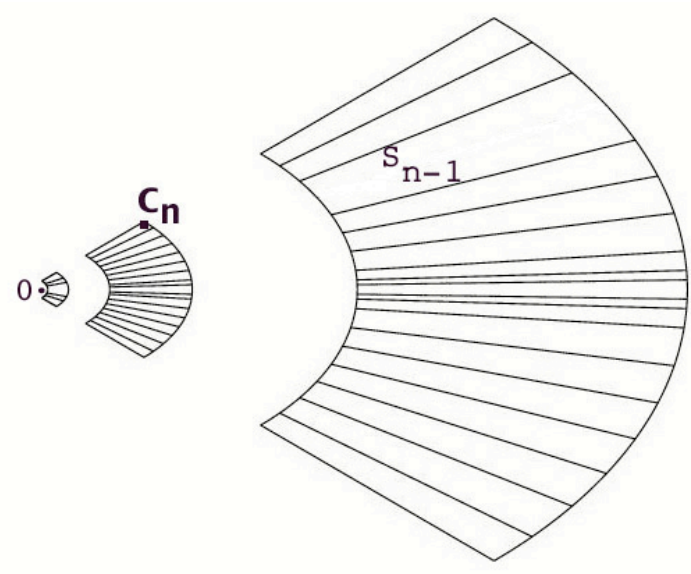

Figure 4. The sectors

Let $C_{n}$ be the upper right corner of the sector $S_{n}:=\left\{z \in \mathbb{C}: \frac{1}{2^{2 n+1}} \leq|z| \leq \frac{1}{2^{2 n}}:|\arg z| \leq \pi / 4\right\}$. Define the functions $f$ and $g$ by $f(z)=z$ and

$$
g(z)= \begin{cases}\bar{C}_{n} & \text { if } z \in S_{n} \\ 0 & \text { if } z=0\end{cases}
$$

Then $f$ and $g$ belong to $A^{1}(K)$ and $|f| \leq|g|$; note that for $z \in S_{n}$

$$
|f(z)| \leq \max _{u \in S_{n}}|u|=2^{-2 n}=\left|C_{n}\right|=|g(z)| .
$$

Since $g^{\prime} \equiv 0$ on $K^{\circ}$, we obtain for every $z \in K, z \neq 0$ :

$$
\Delta(z):=\frac{d}{d z}\left(\frac{f^{2}(z)}{g(z)}\right)=2 f^{\prime}(z) f(z) \frac{1}{g(z)}=2 \frac{z}{g(z)} .
$$

If $z_{n}=C_{n}=r_{n} e^{i \pi / 4}$, then $\Delta\left(z_{n}\right)=2 e^{2 i \pi / 4}$; but if $z_{n}=\bar{C}_{n}$, then $z_{n} \in K$ and $\Delta\left(z_{n}\right)=2$. Thus $\lim _{\substack{z \rightarrow 0 \\ \in K \backslash\{0\}}} \Delta(z)$ does not exist. Hence $f^{2} / g \notin A^{1}(K)$.

In order to study division in the algebras $C^{m}(K)$ and $A^{m}(K), m \geq 3$, we use a variant of the Faà di Bruno formula, given in [4].

Theorem 3.7. - Let

$$
\boldsymbol{M}^{j}=\left\{\boldsymbol{k}=\left(k_{1}, \ldots, k_{j}\right) \in\left(\mathbb{N}^{*}\right)^{j}, k_{1} \geq k_{2} \geq \cdots \geq k_{j} \geq 1\right\}
$$


be the set of ordered multi-indices in $\mathbb{N}^{*}=\{1,2, \ldots\}$. Then for every $f, g \in C^{n}(\mathbb{R})$

$$
(f \circ g)^{(n)}(x)=\sum_{j=1}^{n} f^{(j)}(g(x))\left(\sum_{\substack{\boldsymbol{k} \in \boldsymbol{M}^{j} \\|\boldsymbol{k}|=n}} C_{\boldsymbol{k}}^{n} g^{(\boldsymbol{k})}(x)\right),
$$

where

$$
C_{\boldsymbol{k}}^{n}=\frac{\left(\begin{array}{l}
n \\
\boldsymbol{k}
\end{array}\right)}{\prod_{i} n(\boldsymbol{k}, i) !} .
$$

Here $g^{(\boldsymbol{k})}=g^{\left(k_{1}\right)} g^{\left(k_{2}\right)} \ldots g^{\left(k_{j}\right)},\left(\begin{array}{l}n \\ \boldsymbol{k}\end{array}\right)$ is the multinomial coefficient defined by $\left(\begin{array}{l}n \\ \boldsymbol{k}\end{array}\right)=\frac{n !}{k_{1} ! k_{2} ! \ldots k_{j} !}$, where $|\boldsymbol{k}|:=k_{1}+\cdots+k_{j}=n$, and $n(\boldsymbol{k}, i)$ is the number of times the integer $i$ appears in the $j$-tuple $\boldsymbol{k}\left(i \in \mathbb{N}^{*}\right.$ and $\left.\boldsymbol{k} \in\left(\mathbb{N}^{*}\right)^{j}\right)$.

Theorem 3.8. - Let $f, g \in A^{m}(K)$ and suppose that $|f| \leq|g|$. Then, for every $n \in \mathbb{N}$ with $0 \leq n \leq m$, the following estimate holds on $K^{\circ} \backslash Z(g)$ :

$$
\left(\frac{f^{m+2}}{g}\right)^{(n)} \leq C|g|^{m+1-n}
$$

Proof. - Let $I(z):=1 / z$ and $h(z):=1 / g(z)$. Then $h=I \circ g$. Also,

$$
I^{(j)}(z)=j !(-1)^{j} \frac{1}{z^{j+1}} .
$$

By Theorem 3.7, for $1 \leq \mu \leq m$,

$$
\begin{aligned}
h^{(\mu)}= & (I \circ g)^{(\mu)}=\sum_{j=1}^{\mu}\left(I^{(j)} \circ g\right)\left(\sum_{\substack{\boldsymbol{k} \in \boldsymbol{M}^{j} \\
|\boldsymbol{k}|=\mu}} C_{\boldsymbol{k}}^{\mu} g^{(\boldsymbol{k})}\right) \\
& =\sum_{j=1}^{\mu} j !(-1)^{j} \frac{1}{g^{j+1}}\left(\sum_{\substack{\boldsymbol{k} \in \boldsymbol{M}^{j} \\
|\boldsymbol{k}|=\mu}} C_{\boldsymbol{k}}^{\mu} g^{(\boldsymbol{k})}\right) .
\end{aligned}
$$

We may assume that $\|g\|_{\infty} \leq 1$. Since the derivatives of $g$ are bounded up to the order $m$, we conclude that on $K^{\circ} \backslash Z(g)$

By Leibniz's formula

$$
\left|h^{(\mu)}\right| \leq C\left|\frac{1}{g}\right|^{\mu+1}
$$

$$
\left(f^{m+2} \cdot h\right)^{(n)}=\sum_{\ell=0}^{n}\left(\begin{array}{l}
n \\
\ell
\end{array}\right) h^{(n-\ell)}\left(f^{m+2}\right)^{(\ell)} .
$$


Next we have to estimate the derivatives of $f^{m+2}$. Let $p(z)=z^{m+2}$. Using a second time Theorem 3.7, we obtain for $1 \leq \ell \leq m$,

$$
\left(f^{m+2}\right)^{(\ell)}=(p \circ f)^{(\ell)}=\sum_{j=1}^{\ell}\left(p^{(j)} \circ f\right)\left(\sum_{\substack{\boldsymbol{k} \in M^{j} \\|\boldsymbol{k}|=\ell}} C_{\boldsymbol{k}}^{\ell} f^{(\boldsymbol{k})}\right) .
$$

Since

$$
\left(z^{m+2}\right)^{(j)}=(m+2) \ldots(m+2-j+1) z^{m+2-j},
$$

we estimate as follows (note that $|f| \leq|g| \leq 1$ ):

$$
\left|\left(f^{m+2}\right)^{(\ell)}\right| \leq \sum_{j=1}^{\ell} C_{j}|f|^{m+2-j} \leq \tilde{C}|f|^{m+2-\ell} .
$$

In view of the assumption $|f| \leq|g|$, equation (3.8) then yields

$$
\begin{gathered}
\left|\left(\frac{f^{m+2}}{g}\right)^{(n)}\right| \leq \tilde{C} \sum_{\ell=0}^{n}\left(\begin{array}{c}
n \\
\ell
\end{array}\right)\left|\frac{1}{g}\right|^{n-\ell+1}|f|^{m+2-\ell} \\
\underset{|f| \leq|g|}{\leq} \tilde{C} \sum_{\ell=0}^{n}\left(\begin{array}{l}
n \\
\ell
\end{array}\right)|g|^{m+1-n}=\kappa|g|^{m+1-n} .
\end{gathered}
$$

A similar proof applied to the mixed partial derivatives of $f, g \in C^{m}(K)$ yields an analogous result.

Theorem 3.9. - Let $f, g \in C^{m}(K)$ and suppose that $|f| \leq|g|$. Then, for every $n \in \mathbb{N}$ with $0 \leq n \leq m$, the following estimate holds on $K^{\circ} \backslash Z(g)$ :

$$
D_{n}\left(\frac{f^{m+2}}{g}\right) \leq C|g|^{m+1-n}
$$

where $D_{n}=(\partial x)^{j_{1}}(\partial y)^{j_{2}}$ with $j_{1}+j_{2}=n$.

Theorem 3.10. - Let $K \subseteq \mathbb{C}$ be a compact set. Then the following assertions hold:

a) If $f, g \in A^{m}(K)$ satisfy $|f| \leq|g|$, then $f^{m+2} \in I_{A^{m}(K)}(g)$.

b) If $f, g \in C^{m}(K)$ satisfy $|f| \leq|g|$ and if $Z(g)$ has no cluster points in $K^{\circ}$, then $f^{m+2} \in I_{C^{m}(K)}(g)$.

Proof. - a) Due to holomorphy, the quotient $f^{m+2} / g$ is holomorphic at every isolated zero $z_{0}$ of $g$ in $K^{\circ}$, since $m\left(f, z_{0}\right) \geq m\left(g, z_{0}\right)$, where $m\left(f, z_{0}\right)$ denotes the multiplicity of the zero $z_{0}$. Let

$$
h(z)= \begin{cases}\frac{f^{m+2}(z)}{g(z)} & \text { if } z \in K \backslash Z(g) \\ 0 & \text { if } z \in Z(g) .\end{cases}
$$


Since $h \equiv 0$ on $Z(g)^{\circ}$, the fact that $\partial Z(g)^{\circ} \subseteq \partial K$ implies that $h$ is holomorphic on

$$
K^{\circ} \stackrel{*}{=}\left(K \backslash Z(g)^{\circ}\right)^{\circ} \cup Z(g)^{\circ} .
$$

(* was shown in [5, p. 2219]). We may assume that $|g| \leq 1$ on $K$. By Theorem 3.8,

$$
\left|\left(f^{m+2} / g\right)^{(j)}\right| \leq|g|
$$

on $K^{\circ} \backslash Z(g)$ for every $0 \leq j \leq m$. Since $f, g \in A(K)^{m}$, the derivatives (a priori defined only on $K^{\circ}$ ) are continuously extendable to $K$. We denote them by the usual symbol $f^{(j)}$ etc. Thus $\left|h^{(j)}\right| \leq|g|$ on $K \backslash Z(g)$ and so $\left.h^{(j)}\right|_{K \backslash Z(g)}$ admits a continuous extension to $K$.

b) Similar proof, since we assumed that the zeros of $f$ and $g$ are isolated.

\section{The $f^{N}$-problem in $A(K)$.}

Here we present some sufficient conditions on the generators $f_{j}$ that guarantee that $|g| \leq$ $\sum_{j=1}^{N}\left|f_{j}\right|$ implies that $g^{N} \in I_{A(K)}\left(f_{1}, \ldots, f_{n}\right)$ for some $N \in \mathbb{N}$.

Lemma 4.1. - Let $A=C^{1}(K)$ or $C(K) \cap C^{1}\left(K^{\circ}\right)$. If $g, f_{j} \in A$ satisfy $|g| \leq|\boldsymbol{f}|$, where $\boldsymbol{f}=$ $\left(f_{1}, \ldots, f_{n}\right)$, then $\left.\left(g^{4} /|\boldsymbol{f}|^{2}\right)\right|_{K \backslash Z\left(|\boldsymbol{f}|^{2}\right)}$ admits an extension to an element in $A$ whenever $|\boldsymbol{f}|$ has only isolated zeros in $K^{\circ}$. The power 4 is best possible (within $\mathbb{N}$ ). In particular, $g^{4} \in I_{A}\left(f_{1}, \ldots, f_{n}\right)$.

Proof. - To show that 4 is best possible, consider the function $f(z)=\bar{z}$ and $g(z)=z$. Then $z^{3} /|z|^{2}=z^{2} / \bar{z}$ is not differentiable at 0 (see the example in Theorem 3.6(b)). Now if $D=\partial$ or $\bar{\partial}$, then ${ }^{(4)}$ on $K^{\circ} \backslash Z\left(|\boldsymbol{f}|^{2}\right)$

$$
\begin{aligned}
D\left(\frac{g^{4}}{|\boldsymbol{f}|^{2}}\right) & =\frac{4|\boldsymbol{f}|^{2} g^{3} D g-g^{4}(\boldsymbol{f} \cdot D \overline{\boldsymbol{f}}+D \boldsymbol{f} \cdot \overline{\boldsymbol{f}})}{|\boldsymbol{f}|^{4}} \\
& =4 g\left(\left(\frac{g}{|\boldsymbol{f}|}\right)^{2} D g\right)-\left(\frac{g}{|\boldsymbol{f}|}\right)^{4}(\boldsymbol{f} \cdot D \overline{\boldsymbol{f}}+D \boldsymbol{f} \cdot \overline{\boldsymbol{f}})
\end{aligned}
$$

If $|\boldsymbol{f}(z)|=0$, then $g(z)=0$ and so the boundedness of the term $g /|\boldsymbol{f}|$ yields the continuous extensions of $D\left(\frac{g^{4}}{|\boldsymbol{f}|^{2}}\right)$ with value 0 . Since the zeros of $|\boldsymbol{f}|$ are isolated within $K^{\circ}$, we get from the mean value theorem of the differential calculus, that both partial derivatives exist at those zeros and coincide with these extensions. Hence $\left.\left(g^{4} /|\boldsymbol{f}|^{2}\right)\right|_{K \backslash Z(\boldsymbol{f})}$ admits the desired extension.

By a similar proof we have

Lemma 4.2. - Let $A=C^{1}(K)$ or $C(K) \cap C^{1}\left(K^{\circ}\right)$. If $g, f_{j} \in A$ satisfy $|g|^{2} \leq|\boldsymbol{f}|$, where $\boldsymbol{f}=\left(f_{1}, \ldots, f_{n}\right)$, then $\left.\left(g^{7} /|\boldsymbol{f}|^{2}\right)\right|_{K \backslash Z\left(|\boldsymbol{f}|^{2}\right)}$ admits an extension to an element in $A$ whenever $|\boldsymbol{f}|$ has only isolated zeros in $K^{\circ}$. In particular, $g^{7} \in I_{A}\left(f_{1}, \ldots, f_{n}\right)$.

We don't know whether the power 7 is optimal.

Now we use again the convenient matricial notation from section 2.

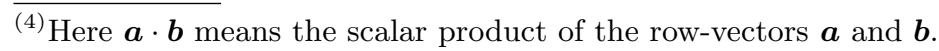


Proposition 4.3. - Suppose that $\boldsymbol{f}=\left(f_{1}, \ldots, f_{n}\right) \in A(K)^{n}$ and that $g \in A(K)$ satisfies $|g| \leq$ $\sum_{j=1}^{n}\left|f_{j}\right|$. If there is a solution $\boldsymbol{x}$ in $C_{\bar{\partial}, 1}(K)$ to $\boldsymbol{x} \boldsymbol{f}^{t}=g$, then there exists $\boldsymbol{u}=\left(u_{1}, \ldots, u_{n}\right) \in$ $A(K)^{n}$ such that $\boldsymbol{u} \boldsymbol{f}^{t}=g^{5}$ whenever $|\boldsymbol{f}|$ has only isolated zeros in $K^{\circ}$ and there exists $\boldsymbol{v}=$ $\left(v_{1}, \ldots, v_{n}\right) \in A(K)^{n}$ such that $\boldsymbol{v} \boldsymbol{f}^{t}=g^{6}$ whenever $\boldsymbol{f} \in A(K)^{n}$ is arbitrary.

Proof. - We first suppose that $Z(|\boldsymbol{f}|)$ does not admit a cluster point within $K^{\circ}$. As in Theorem 2.2, we consider on $K \backslash Z(|\boldsymbol{f}|)$ the matrix

$$
F=\left(\left(\bar{\partial} \boldsymbol{x}^{t} \cdot \overline{\boldsymbol{f}}\right)^{t}-\bar{\partial} \boldsymbol{x}^{t} \cdot \overline{\boldsymbol{f}}\right) \frac{1}{|\boldsymbol{f}|^{2}}
$$

Using the facts that $\boldsymbol{x} \in C_{\overline{\mathrm{D}}, 1}(K)^{n}$ and $g^{4} /|\boldsymbol{f}|^{2} \in C(K) \cap C^{1}\left(K^{\circ}\right)$ we conclude from Lemma 4.1 that $F g^{4}$ extends to an antisymmetric matrix over $C(K) \cap C^{1}\left(K^{\circ}\right)$. Thus, by Theorem 2.1, the system $\bar{\partial} H=F g^{4}$ admits a matrix solution $H$ over $C(K) \cap C^{1}\left(K^{\circ}\right)$. Note that $H$ can be chosen to be antisymmetric, too. Now let

$$
\boldsymbol{u}=g^{4} \boldsymbol{x}-\boldsymbol{f} H .
$$

Then $\boldsymbol{u} \in C(K)^{n} \cap\left(C^{1}\left(K^{\circ}\right)\right)^{n}$. Moreover, on $K^{\circ} \backslash Z(|\boldsymbol{f}|), \bar{\partial} \boldsymbol{u}=0$ because

$$
\begin{aligned}
\bar{\partial} \boldsymbol{u} & =g^{4} \bar{\partial} \boldsymbol{x}-\boldsymbol{f} \cdot \bar{\partial} H=g^{4} \bar{\partial} \boldsymbol{x}-\boldsymbol{f} \cdot\left(\overline{\boldsymbol{f}}^{t} \cdot \bar{\partial} \boldsymbol{x}-\bar{\partial} \boldsymbol{x}^{t} \cdot \overline{\boldsymbol{f}}\right) \frac{g^{4}}{|\boldsymbol{f}|^{2}} \\
& =g^{4} \frac{\left(\boldsymbol{f} \cdot \bar{\partial} \boldsymbol{x}^{t}\right) \overline{\boldsymbol{f}}}{|\boldsymbol{f}|^{2}}=g^{4} \frac{\left(\bar{\partial}\left(\boldsymbol{f} \cdot \boldsymbol{x}^{t}\right)\right) \overline{\boldsymbol{f}}}{|\boldsymbol{f}|^{2}}=g^{4} \frac{(\bar{\partial} g) \overline{\boldsymbol{f}}}{|\boldsymbol{f}|^{2}}=\mathbf{0} .
\end{aligned}
$$

Since it is assumed that every point in $Z(|\boldsymbol{f}|) \cap K^{\circ}$ is an isolated point, the continuity of $\bar{\partial} \boldsymbol{u}$ implies that $\bar{\partial} \boldsymbol{u}=0$ on $K^{\circ}$. Hence $\boldsymbol{u} \in A(K)^{n}$. By antisymmetry, ${ }^{(5)} \boldsymbol{f} H \boldsymbol{f}^{t}=0$ and so

$$
\boldsymbol{u} \boldsymbol{f}^{t}=g^{4} \boldsymbol{x} \boldsymbol{f}^{t}-\boldsymbol{f} H \boldsymbol{f}^{t}=g^{4} g=g^{5}
$$

Now let $\boldsymbol{f} \in A(K)^{n}$ be arbitrary. If $|\boldsymbol{f}|$ is identically zero, then nothing is to prove. Hence we may assume that $S:=K \backslash Z(|\boldsymbol{f}|)^{\circ} \neq \emptyset$. By the first case, there is $\boldsymbol{u} \in A(S)^{n}$ such that $\boldsymbol{u} \boldsymbol{f}^{t}=g^{5}$ on $S$. Since $\partial\left(Z(|f|)^{\circ}\right) \subseteq \partial K$, we again have

$$
S^{\circ} \cup Z(|f|)^{\circ}=K^{\circ}
$$

Hence the vector-valued function

$$
\boldsymbol{v}(z)= \begin{cases}g(z) \boldsymbol{u}(z) & \text { if } z \in S \\ \mathbf{0} & \text { if } z \in K \backslash S\end{cases}
$$

is well defined, continuous on $K$ and each of its coordinates is holomorphic on $K^{\circ}$. It easily follows that $\boldsymbol{v} \boldsymbol{f}^{t}=g^{6}$.

$\left.\overline{{ }^{(5)} \boldsymbol{f} H \boldsymbol{f}^{t}=(\boldsymbol{f} H} \boldsymbol{f}^{t}\right)^{t}=\boldsymbol{f} H^{t} \boldsymbol{f}^{t}=-\boldsymbol{f} H \boldsymbol{f}^{t}$ 
According to [3], an ideal $I$ in a uniform algebra $A$ is said to have the Forelli property (say $I \in \mathscr{F})$, if there exists $f \in I$ with $Z(f)=Z(I):=\bigcap_{h \in I} Z(h)$. It is obvious that in $C(K)$ every finitely generated ideal has the Forelli-property; just consider the function

$$
f=\sum_{j=1}^{n}\left|f_{j}\right|^{2}=\sum_{j=1}^{n} \bar{f}_{j} f_{j} \in I_{C(K)}\left(f_{1}, \ldots, f_{n}\right) .
$$

It was shown in $[\mathbf{3}]$ that there exist finitely generated ideals in the disk-algebra that do not have this property. A natural question, therefore, is whether $C_{\bar{\partial}, 1}(K)$ has the Forelli-property. A sufficient condition for $I=I\left(f_{1}, \ldots, f_{n}\right)$ to belong to $\mathscr{F}$ is that there exists $h \in I$ such that ${ }^{(6)}$

$$
|h| \geq \sum_{j=1}^{n}\left|f_{j}\right|^{2} .
$$

Theorem 4.4. - Let $\boldsymbol{f}=\left(f_{1}, \ldots, f_{n}\right) \in A(K)^{n}$ and suppose that $|\boldsymbol{f}|$ has only isolated zeros in $K^{\circ}$. We assume that there are $h_{j} \in C_{\bar{\partial}, 1}(K)$ such that

$$
\left|\sum_{j=1}^{n} h_{j} f_{j}\right| \geq \sum_{j=1}^{n}\left|f_{j}\right|^{2} .
$$

Then for every $g \in A(K)$ satisfying $|g| \leq \sum_{j=1}^{n}\left|f_{j}\right|$ we have $g^{12} \in I_{A(K)}\left(f_{1}, \ldots, f_{n}\right)$.

Proof. - Let $h=\sum_{j=1}^{n} h_{j} f_{j}$. Then $|g|^{2} \leq n \sum_{j=1}^{n}\left|f_{j}\right|^{2} \leq n|h|$. By Proposition 3.6, there is $k \in C_{\bar{\partial}, 1}(K)$ such that $g^{8}=k h$. Hence

$$
g^{8}=\sum_{j=1}^{n}\left(k h_{j}\right) f_{j} \in I_{C_{\bar{\partial}, 1}(K)}\left(f_{1}, \ldots, f_{n}\right) .
$$

Since $|g| \leq \sqrt{n}|\boldsymbol{f}|$ implies that $g^{4} /|\boldsymbol{f}|^{2} \in C(K) \cap C^{1}\left(K^{\circ}\right)$ (Proposition 4.1), we obtain in a similar manner as in Proposition 4.3 that $g^{12} \in I_{A(K)}\left(f_{1}, \ldots, f_{n}\right)$ (just consider the data $\bar{\partial} H=F g^{4}$, $\boldsymbol{x}=\left(k h_{1}, \ldots, k h_{n}\right), \boldsymbol{u}=g^{4} \boldsymbol{x}-\boldsymbol{f} H$, and use the fact that $\boldsymbol{x} \boldsymbol{f}^{t}=g^{8}$ in order to get $\left.u \boldsymbol{f}^{t}=g^{12}\right)$.

\section{The $f^{N}$-problem in $C(K)$ and $C^{1}(K)$}

Proposition 5.1. - Let $h, f_{j} \in C(K)$ satisfy $|h| \leq \sum_{j=1}^{n}\left|f_{j}\right|$. Then $h^{2} \in I_{C(K)}\left(f_{1}, \ldots, f_{n}\right)$. Within $\mathbb{N}$, the constant 2 is best possible.

Proof. - Consider on $K \backslash \bigcap_{k=1}^{n} Z\left(f_{k}\right)$ the functions

$$
q_{j}=\frac{h \bar{f}_{j}}{\sum_{k=1}^{n}\left|f_{k}\right|^{2}} .
$$

\footnotetext{
${ }^{(6)}$ Instead of 2 we may of course take any power $\alpha>0$.
} 
Then, by Cauchy-Schwarz,

$$
\left|q_{j}\right| \leq \frac{\left(\sum_{k=1}^{n}\left|f_{k}\right|\right)^{2}}{|\boldsymbol{f}|^{2}} \leq n
$$

Hence $q_{j}$ is bounded on $K \backslash \bigcap_{k=1}^{n} Z\left(f_{k}\right)$. It is then clear that $g_{j}:=h q_{j} \in C(K)$ and that

$$
\sum_{j=1}^{n} g_{j} f_{j}=h^{2}
$$

By Theorem 3.6(a), the power $n=2$ is best possible.

Proposition 5.2. - Let $h, f_{j} \in C^{1}(K)$ satisfy $|h| \leq \sum_{j=1}^{n}\left|f_{j}\right|$. If we suppose that $\bigcap_{j=1}^{n} Z\left(f_{j}\right) \cap K^{\circ}$ is discrete, then $h^{3} \in I_{C^{1}(K)}\left(f_{1}, \ldots, f_{n}\right)$. Within $\mathbb{N}$, the constant 3 is best possible.

Proof. - The example $h(z)=z$ and $f(z)=\bar{z}$ in Proposition 3.6 (b) shows that 3 is best possible. On $K \backslash \bigcap_{j=1}^{n} Z\left(f_{j}\right)$, let

$$
q_{j}=\frac{\bar{f}_{j} h^{3}}{\sum_{k=1}^{n}\left|f_{k}\right|^{2}}
$$

We claim that $q_{j}$ has an extension to a function in $C^{1}(K)$. Let $\boldsymbol{f}=\left(f_{1}, \ldots, f_{n}\right)$. Since outside $\bigcap_{j=1}^{n} Z\left(f_{j}\right)$

$$
\left|q_{j}\right| \leq \frac{|\boldsymbol{f}|\left|h^{3}\right|}{|\boldsymbol{f}|^{2}}=\left(\frac{|h|}{|\boldsymbol{f}|}\right)|h|^{2} \leq C|\boldsymbol{f}|^{2}
$$

we immediately see that $q_{j}$ can be continuously extended to $K$ with value 0 on $Z(|\boldsymbol{f}|)$. Now, if $D=\partial$ or $\bar{\partial}$, then on $K^{\circ} \backslash \bigcap_{j=1}^{n} Z\left(f_{j}\right)=K^{\circ} \backslash Z(|\boldsymbol{f}|)$

$$
D q_{j}=\frac{|\boldsymbol{f}|^{2}\left(\left(D \bar{f}_{j}\right) h^{3}+3 \bar{f}_{j}(D h) h^{2}\right)-\bar{f}_{j} h^{3}(\boldsymbol{f} D \overline{\boldsymbol{f}}+\overline{\boldsymbol{f}} D \boldsymbol{f})}{|\boldsymbol{f}|^{4}} .
$$

Using that the derivatives are continuous and that $\max \left\{|h|,\left|f_{j}\right|\right\} \leq \kappa|\boldsymbol{f}|$, we obtain constants $C_{j}$ such that

$$
\begin{gathered}
\left|D q_{j}\right| \leq\left(\frac{|h|}{|\boldsymbol{f}|}\right)^{2}\left|D \bar{f}_{j}\right||h|+3|\boldsymbol{f}||D h|\left(\frac{|h|}{|\boldsymbol{f}|}\right)^{2}+\frac{|\boldsymbol{f}||h|^{3}|\boldsymbol{f}|(|D \boldsymbol{f}|+|D \overline{\boldsymbol{f}}|)}{|\boldsymbol{f}|^{4}} \\
\leq C_{1}|\boldsymbol{f}|+C_{2}|\boldsymbol{f}|+|\boldsymbol{f}|(|D \boldsymbol{f}|+|D \overline{\boldsymbol{f}}|) \leq C_{3}|\boldsymbol{f}|
\end{gathered}
$$

Thus the partial derivatives admit a continuous extension to $K$. Since at an isolated zero of $|\boldsymbol{f}|$ the partial derivatives of $q_{j}$ exist by the mean value theorem in differential calculus, we are done. 


\section{Questions}

In this final section we would like to ask several questions and present a series of problems.

1) Let $f, f_{1}, \ldots, f_{n} \in A(\overline{\mathbb{D}})$ satisfy $|f| \leq \sum_{j=1}^{n}\left|f_{j}\right|$. Is $f^{3} \in I_{A(\overline{\mathbb{D}})}\left(f_{1}, \ldots, f_{n}\right)$ ?

2 ) Is there a simple example of a triple $\left(f, f_{1}, f_{2}\right)$ in $A(\mathbb{D})$ such that $|f| \leq\left|f_{1}\right|+\left|f_{2}\right|$, but for which $f^{2} \notin I_{A(\overline{\mathbb{D}})}\left(f_{1}, f_{2}\right)$ ?

3 ) Is there a simple example of a function $f$, continuous on $\overline{\mathbb{D}}$, such that the Pompeiu-integral

$$
P_{f}(z)=\iint_{\overline{\mathbb{D}}} \frac{f(\xi)}{\xi-z} d \sigma(\xi)
$$

is not continuously differentiable in $\mathbb{D}$ ?

Let $M$ and $S$ be two specific classes of functions on the unit disk. For example $M$ and $S$ coincide with $C_{b}(\mathbb{D})$, the set of all bounded, continuous and complex-valued functions on $\mathbb{D} ; C_{b}^{1}(\mathbb{D})=$ $C_{b}(\mathbb{D}) \cap C^{1}(\mathbb{D})$; or $C_{b b}^{1}(\mathbb{D})$, the set of all $C^{1}$-functions $u$ on $\mathbb{D}$ for which $u$ and $\nabla u$ are bounded.

Give necessary and sufficient conditions on $f \in M$ such that the $\bar{\partial}$-equation $\bar{\partial} u=f$ admits a solution $u \in S$ :

4) $f \in C_{b}^{1}(\mathbb{D}), u \in C_{b}^{1}(\mathbb{D})$;

5) $f \in C_{b}^{1}(\mathbb{D}), u \in C(\overline{\mathbb{D}}) \cap C^{1}(\mathbb{D})$;

6) $f \in C_{b}^{1}(\mathbb{D}), u \in C(\overline{\mathbb{D}}) \cap C_{b b}^{1}(\mathbb{D})$;

7) $f \in C^{1}(\mathbb{D}), u \in C^{1}(\mathbb{D})$;

8) $f \in C^{1}(\mathbb{D}), u \in C_{b}^{1}(\mathbb{D})$;

9) $f \in C^{1}(\mathbb{D}), u \in C(\overline{\mathbb{D}}) \cap C^{1}(\mathbb{D})$;

10) $f \in C^{1}(\mathbb{D}), u \in C(\overline{\mathbb{D}}) \cap C_{b b}^{1}(\mathbb{D})$;

11) $f \in C(\overline{\mathbb{D}}), u \in C^{1}(\mathbb{D})$;

12) $f \in C(\overline{\mathbb{D}}), u \in C_{b}^{1}(\mathbb{D})$;

13) $f \in C(\overline{\mathbb{D}}), u \in C(\overline{\mathbb{D}}) \cap C^{1}(\mathbb{D})$;

14) $f \in C(\overline{\mathbb{D}}), u \in C(\overline{\mathbb{D}}) \cap C_{b b}^{1}(\mathbb{D})$.

\section{References}

[1] M. Andersson, Topics in Complex Analysis, Springer, New York 1997. 4, 5

[2] J.B. Garnett, Bounded Analytic Functions, Academic Press, New York, 1981. 2

[3] R. Mortini, The Forelli problem concerning ideals in the disk algebra $A(\mathbf{D})$, Proc. Amer. Math. Soc. 95 (1985), 261-264. 20

[4] R. Mortini, The Faà di Bruno formula revisited, to appear in Elem. Math. 2013. 15

[5] R. Mortini, R. Rupp, The Bass stable rank for the real Banach algebra $A(K)_{s y m}$, J. Funct. Anal. 261 (2011), 2214-2237. 18

[6] R. Mortini, R. Rupp, A solution to the Bézout equation in $A(K)$ without Gelfand theory. Archiv Math. 99 (2012), 49-59. 2, 5 
[7] R. Mortini, B. Wick, Simultaneous stabilization in $A_{\mathbb{R}}(\mathbb{D})$, Studia Math. 191 (2009), 223-235. 5

[8] R. Narasimhan Complex variables in one variable, Birkhäuser, Boston, 1985. 5

[9] W. Rudin Functional Analysis, Second Edition, MacGraw-Hill, 1991. 4

Raymond Mortini, Université de Lorraine, Département de Mathématiques et Institut Élie Cartan de Lorraine, UMR 7502, Ile du Saulcy, F-57045 Metz, France • E-mail : mortini@univ-metz.fr

Rudolf Rupp, Fakultät Allgemeinwissenschaften, Georg-Simon-Ohm-Hochschule Nürnberg, Kesslerplatz 12, D-90489 Nürnberg, Germany • E-mail : Rudolf.Rupp@ohm-hochschule.de 